Supporting Information

\title{
Recommender system of successful processing conditions for new compounds based on a parallel experimental dataset
}

Hiroyuki Hayashi ${ }^{1,2,3}$, Katsuyuki Hayashi ${ }^{1}, K_{\text {eita Kouzai }}{ }^{1}$, Atsuto Seko ${ }^{1,2,3}$, and Isao Tanaka ${ }^{1,3}$

1. Department of Materials Science and Engineering, Kyoto University, Sakyo, Kyoto 606-8501, Japan

2. JST, PRESTO, Kawaguchi 332-0012, Japan

3. Center for Materials Research by Information Integration, National Institute for Materials Science, Tsukuba 305-0047, Japan 
Table S1. Starting materials used as cation sources for four methods.

\begin{tabular}{|c|c|c|c|c|c|}
\hline Element & Starting materials & SSR & CES & $\mathrm{PC}$ & $\mathrm{SC}$ \\
\hline \multirow{3}{*}{$\mathrm{Al}$} & $\mathrm{Al}_{2} \mathrm{O}_{3}$ & $\checkmark$ & & & \\
\hline & $\mathrm{AlCl}_{3} 6 \mathrm{H}_{2} \mathrm{O}$ & & $\checkmark$ & $\checkmark$ & $\checkmark$ \\
\hline & $\mathrm{Al}\left(\mathrm{NO}_{3}\right)_{3}$ & & $\checkmark$ & & \\
\hline \multirow{2}{*}{$\mathrm{Ba}$} & $\mathrm{BaCl}_{2}$ & & $\checkmark$ & $\checkmark$ & $\checkmark$ \\
\hline & $\mathrm{BaO}$ & $\checkmark$ & & & \\
\hline \multirow{2}{*}{$\mathrm{Bi}$} & $\mathrm{Bi}_{2} \mathrm{O}_{3}$ & $\checkmark$ & & & \\
\hline & $\mathrm{BiCl}_{3}$ & & $\checkmark$ & $\checkmark$ & $\checkmark$ \\
\hline \multirow{3}{*}{$\mathrm{La}$} & $\mathrm{La}_{2} \mathrm{O}_{3}$ & $\checkmark$ & & & \\
\hline & $\mathrm{LaCl}_{3} 7 \mathrm{H}_{2} \mathrm{O}$ & & $\checkmark$ & $\checkmark$ & $\checkmark$ \\
\hline & $\mathrm{La}\left(\mathrm{NO}_{3}\right)_{3}$ & & $\checkmark$ & & \\
\hline \multirow{4}{*}{ Mo } & $\mathrm{MoCl}_{5}$ & & $\checkmark$ & & $\checkmark$ \\
\hline & $\mathrm{MoO}_{3}$ & $\checkmark$ & & & \\
\hline & $\left(\mathrm{NH}_{4}\right)_{6} \mathrm{Mo}_{7} \mathrm{O}_{24} 4 \mathrm{H}_{2} \mathrm{O}$ & & & $\checkmark$ & $\checkmark$ \\
\hline & peroxo-Mo & & $\checkmark$ & & \\
\hline \multirow{2}{*}{$\mathrm{Sb}$} & $\mathrm{Sb}_{2} \mathrm{O}_{3}$ & $\checkmark$ & & & \\
\hline & $\mathrm{SbCl}_{3}$ & & $\checkmark$ & $\checkmark$ & $\checkmark$ \\
\hline \multirow{2}{*}{$\mathrm{Sc}$} & $\mathrm{Sc}_{2} \mathrm{O}_{3}$ & $\checkmark$ & & & \\
\hline & $\mathrm{ScCl}_{3}$ & & $\checkmark$ & $\checkmark$ & $\checkmark$ \\
\hline \multirow{2}{*}{$\mathrm{Ti}$} & peroxo-Ti & & $\checkmark$ & $\checkmark$ & $\checkmark$ \\
\hline & $\mathrm{TiO}_{2}$ & $\checkmark$ & & & \\
\hline \multirow{3}{*}{ V } & $\mathrm{NH}_{4} \mathrm{VO}_{3}$ & & & $\checkmark$ & $\checkmark$ \\
\hline & peroxo-V & & $\checkmark$ & & \\
\hline & $\mathrm{V}_{2} \mathrm{O}_{5}$ & $\checkmark$ & & & \\
\hline
\end{tabular}


Table S2. The correspondence between the categorical data "cation mixing ratio" and the cation fraction of ICSD/ICDD compositions.

\begin{tabular}{|c|c|c|c|}
\hline \multirow{2}{*}{$\begin{array}{c}\text { "Cation mixing ratio" } \\
0: 1\end{array}$} & \multicolumn{3}{|c|}{$\begin{array}{l}\text { Cation fraction of ICSD and ICDD } \\
\text { compositions, } x\end{array}$} \\
\hline & & $x=$ & 0.00 \\
\hline $1: 16$ & 0.00 & $<x \leq$ & 0.04 \\
\hline $1: 9$ & 0.04 & $<x \leq$ & 0.07 \\
\hline $1: 8$ & 0.07 & $<x \leq$ & 0.11 \\
\hline $1: 5$ & 0.11 & $<x \leq$ & 0.15 \\
\hline $1: 4$ & 0.15 & $<x \leq$ & 0.19 \\
\hline $2: 7$ & 0.19 & $<x \leq$ & 0.22 \\
\hline $1: 3$ & 0.22 & $<x \leq$ & 0.26 \\
\hline $2: 5$ & 0.26 & $<x \leq$ & 0.30 \\
\hline $1: 2$ & 0.30 & $<x \leq$ & 0.33 \\
\hline $3: 5$ & 0.33 & $<x \leq$ & 0.37 \\
\hline $2: 3$ & 0.37 & $<x \leq$ & 0.41 \\
\hline $3: 4$ & 0.41 & $<x \leq$ & 0.44 \\
\hline $6: 7$ & 0.44 & $<x \leq$ & 0.48 \\
\hline $1: 1$ & 0.48 & $<x \leq$ & 0.52 \\
\hline $7: 6$ & 0.52 & $<x \leq$ & 0.56 \\
\hline $4: 3$ & 0.56 & $<x \leq$ & 0.59 \\
\hline $3: 2$ & 0.59 & $<x \leq$ & 0.63 \\
\hline $5: 3$ & 0.63 & $<x \leq$ & 0.67 \\
\hline $2: 1$ & 0.67 & $<x \leq$ & 0.70 \\
\hline $5: 2$ & 0.70 & $<x \leq$ & 0.74 \\
\hline $3: 1$ & 0.74 & $<x \leq$ & 0.78 \\
\hline $7: 2$ & 0.78 & $<x \leq$ & 0.81 \\
\hline $4: 1$ & 0.81 & $<x \leq$ & 0.85 \\
\hline $5: 1$ & 0.85 & $<x \leq$ & 0.89 \\
\hline $8: 1$ & 0.89 & $<x \leq$ & 0.93 \\
\hline $9: 1$ & 0.93 & $<x \leq$ & 0.96 \\
\hline $16: 1$ & 0.96 & $<x<$ & 1.00 \\
\hline 1:0 & & $x=$ & 1.00 \\
\hline
\end{tabular}


Figure S1. The number of successful and unsuccessful conditions in each synthesis method.

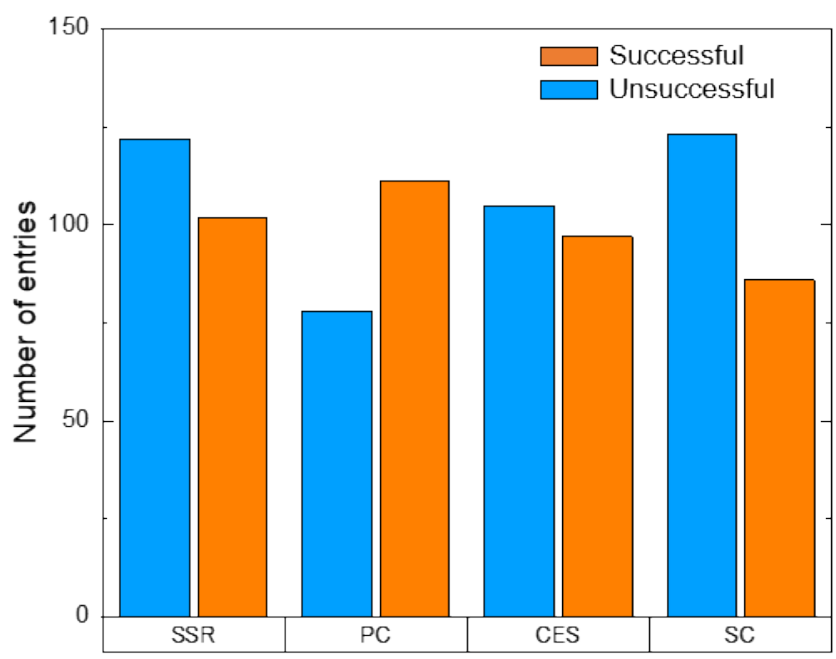


Figure S2. The heatmap of the prediction ability, $p=\sum_{i=\min }^{\max }\left(r_{i}-r_{\mathrm{ave}}\right)^{3} i$ on the core-tensor ranks. Here, $i$ is the center of each bin of the standardized predicted score, $\widehat{y_{s t d}}, \min$ and $\max$ are minimum and maximum values of $i$, respectively, $r_{i}$ is fraction of successful conditions, and $r_{\text {ave }}$ is the average of $r_{i}$, namely 0.48 . The bin size is set at 0.1 . In order to emphasize the difference between the distribution of the successful and unsuccessful conditions, we have used the cube of the fraction of successful conditions. We optimized the ranks through a grid search from the combination of $(2,3,4,5,6,7,8,9,10,11,12,13,14,15)$ on the axes of the "starting material \#1" and "starting material \#2", $(3,5,7,9,11,13,15)$ on "cation mixing ratio", $(2,3)$ on "synthesis method", and $(2,3,4)$ on the "firing temperature". The ranks of the core tensor were thereby selected to be $(6,6,7,2,2)$.

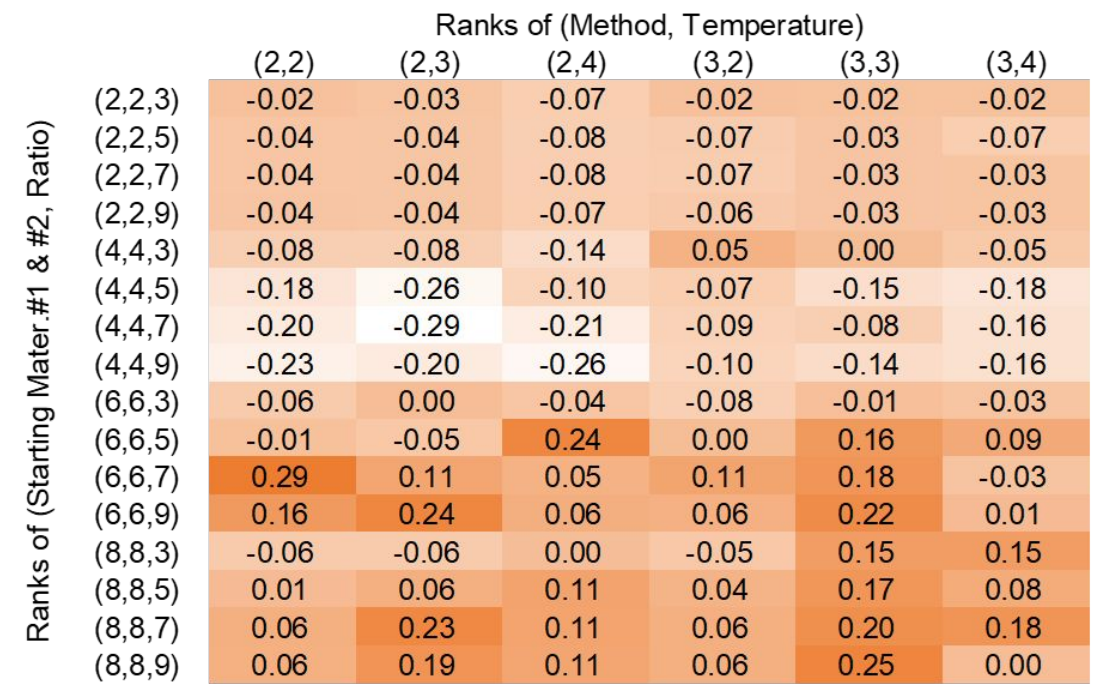


Figure S3. 17 compositions with firing temperatures registered in ICDD and a maximum $\widehat{y_{\text {std }}} \geq 0.05$. These compositions were not used for training data in this study. Check marks were added if there were reported firing temperatures within $\pm 50 \mathrm{~K}$ of firing temperature. The reported firing temperatures of some oxides without check marks are outside the temperature range which we used in the present study. The results of synthesis experiments on these compositions under the synthesis conditions based on the present recommender system are shown in Fig. 9 of the main article.

\begin{tabular}{|c|c|c|c|c|c|c|}
\hline \multirow{2}{*}{$\begin{array}{c}\text { Target } \\
\text { composition }\end{array}$} & \multicolumn{5}{|c|}{ Firing Temperature (K) } & \\
\hline & 873 & 973 & 1073 & 1173 & 1273 & \\
\hline $\mathrm{Ba}_{2} \mathrm{Al}_{2} \mathrm{O}_{5}$ & $\checkmark$ & $\checkmark$ & $\checkmark$ & $\checkmark$ & $\checkmark$ & \\
\hline $\mathrm{Sc}_{2} \mathrm{Ti}_{2} \mathrm{O}_{7}$ & & & & & & \\
\hline $\mathrm{V}_{2} \mathrm{Mo}_{2} \mathrm{O}_{11}$ & $\checkmark$ & & & & & \\
\hline $\mathrm{Ba}_{2} \mathrm{Mo}_{5} \mathrm{O}_{17}$ & $\checkmark$ & & & & & 2 \\
\hline $\mathrm{SbVO}_{4}$ & & $\checkmark$ & $\checkmark$ & & & \\
\hline $\mathrm{Ba}_{2} \mathrm{La}_{2} \mathrm{O}_{5}$ & & & & & $\checkmark$ & 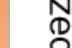 \\
\hline $\mathrm{Ba}_{2} \mathrm{Al}_{10} \mathrm{O}_{17}$ & & & & & & \\
\hline $\mathrm{Ba}_{4} \mathrm{~V}_{2} \mathrm{O}_{9}$ & & $\checkmark$ & & & & 7 \\
\hline $\mathrm{Bi}_{2} \mathrm{La}_{4} \mathrm{O}_{9}$ & & & & & $\checkmark$ & \\
\hline $\mathrm{Sb}_{4} \mathrm{~V}_{2} \mathrm{O}_{11}$ & $\checkmark$ & $\checkmark$ & & & & \\
\hline $\mathrm{Ba}_{2} \mathrm{Sc}_{2} \mathrm{O}_{5}$ & & & & & & ח \\
\hline $\mathrm{Bi}_{2} \mathrm{Ti}_{2} \mathrm{O}_{7}$ & $\checkmark$ & & & & & \\
\hline $\mathrm{Bi}_{3} \mathrm{VO}_{7}$ & & $\checkmark$ & $\checkmark$ & & & \\
\hline $\mathrm{TiMoO}_{5}$ & & $\checkmark$ & & & & 2 \\
\hline $\mathrm{BaBi}_{2} \mathrm{O}_{4}$ & & & $\checkmark$ & & & \\
\hline $\mathrm{La}_{3} \mathrm{Sb}_{5} \mathrm{O}_{12}$ & & & & $\checkmark$ & & \\
\hline $\mathrm{Ba}_{4} \mathrm{~V}_{6} \mathrm{O}_{19}$ & & & & $\checkmark$ & & 0 \\
\hline
\end{tabular}


Table S3. The synthesis conditions of unexperimented target-compositions with the $\widehat{y_{\text {std }}}(i) \geq$ 0.3. The compositions shown in red are registered in ICDD. Check and cross marks in the "Synthesis" column indicate successful and unsuccessful synthesis results obtained in this study, respectively. A minus mark was given when no synthesis experiment was carried out.

\begin{tabular}{|c|c|c|c|c|c|c|c|}
\hline$\widehat{y_{\text {std }}}(i)$ & Target composition & Starting material \#1 & Starting material \#2 & Cation mixing ratio & Method & Firing Temperature $(\mathrm{K})$ & Synthesis \\
\hline 0.74 & $\mathrm{Ba}_{2} \mathrm{Al}_{2} \mathrm{O}_{5}$ & $\mathrm{BaCl}_{2}$ & $\mathrm{AlCl}_{3} 6 \mathrm{H}_{2} \mathrm{O}$ & $1: 1$ & $\mathrm{SC}$ & 973 & $\checkmark$ \\
\hline 0.70 & $\mathrm{Ba}_{2} \mathrm{Al}_{2} \mathrm{O}_{5}$ & $\mathrm{BaCl}_{2}$ & $\mathrm{AlCl}_{3} 6 \mathrm{H}_{2} \mathrm{O}$ & $1: 1$ & $\mathrm{SC}$ & 1073 & $\checkmark$ \\
\hline 0.69 & $\mathrm{Ba}_{2} \mathrm{Al}_{2} \mathrm{O}_{5}$ & $\mathrm{BaCl}_{2}$ & $\mathrm{AlCl}_{3} 6 \mathrm{H}_{2} \mathrm{O}$ & $1: 1$ & $\mathrm{SC}$ & 873 & $\checkmark$ \\
\hline 0.65 & $\mathrm{Ba}_{2} \mathrm{Al}_{2} \mathrm{O}_{5}$ & $\mathrm{BaCl}_{2}$ & $\mathrm{AlCl}_{3} 6 \mathrm{H}_{2} \mathrm{O}$ & $1: 1$ & $\mathrm{SC}$ & 1173 & $\checkmark$ \\
\hline 0.53 & $\mathrm{Ba}_{2} \mathrm{Al}_{2} \mathrm{O}_{5}$ & $\mathrm{BaCl}_{2}$ & $\mathrm{AlCl}_{3} 6 \mathrm{H}_{2} \mathrm{O}$ & $1: 1$ & $\mathrm{SC}$ & 1273 & $\checkmark$ \\
\hline 0.53 & $\mathrm{Sc}_{2} \mathrm{Mo}_{2} \mathrm{O}_{9}$ & $\left(\mathrm{NH}_{4}\right)_{6} \mathrm{Mo}_{7} \mathrm{O}_{24} 4 \mathrm{H}_{2} \mathrm{O}$ & $\mathrm{ScCl}_{3}$ & $1: 1$ & $\mathrm{PC}$ & 873 & - \\
\hline 0.40 & $\mathrm{Al}_{2} \mathrm{MoO}_{6}$ & $\mathrm{AlCl}_{3} 6 \mathrm{H}_{2} \mathrm{O}$ & $\left(\mathrm{NH}_{4}\right)_{6} \mathrm{Mo}_{7} \mathrm{O}_{24} 4 \mathrm{H}_{2} \mathrm{O}$ & $2: 1$ & $\mathrm{PC}$ & 1273 & $\times$ \\
\hline 0.38 & $\mathrm{Al}_{2} \mathrm{MoO}_{6}$ & $\mathrm{AlCl}_{3} 6 \mathrm{H}_{2} \mathrm{O}$ & $\left(\mathrm{NH}_{4}\right)_{6} \mathrm{Mo}_{7} \mathrm{O}_{24} 4 \mathrm{H}_{2} \mathrm{O}$ & $2: 1$ & $\mathrm{PC}$ & 1173 & - \\
\hline 0.37 & $\mathrm{Al}_{2} \mathrm{MoO}_{6}$ & $\mathrm{AlCl}_{3} 6 \mathrm{H}_{2} \mathrm{O}$ & $\left(\mathrm{NH}_{4}\right)_{6} \mathrm{Mo}_{7} \mathrm{O}_{24} 4 \mathrm{H}_{2} \mathrm{O}$ & $2: 1$ & $\mathrm{PC}$ & 1073 & - \\
\hline 0.36 & $\mathrm{Sc}_{2} \mathrm{Mo}_{2} \mathrm{O}_{9}$ & $\mathrm{ScCl}_{3}$ & $\left(\mathrm{NH}_{4}\right)_{6} \mathrm{Mo}_{7} \mathrm{O}_{24} 4 \mathrm{H}_{2} \mathrm{O}$ & $1: 1$ & PC & 973 & - \\
\hline 0.35 & $\mathrm{Sc}_{2} \mathrm{Ti}_{2} \mathrm{O}_{7}$ & $\mathrm{ScCl}_{3}$ & peroxo-Ti & $1: 1$ & $\mathrm{PC}$ & 1273 & $\checkmark$ \\
\hline 0.34 & $\mathrm{Sc}_{2} \mathrm{Mo}_{4} \mathrm{O}_{15}$ & $\left(\mathrm{NH}_{4}\right)_{6} \mathrm{Mo}_{7} \mathrm{O}_{24} 4 \mathrm{H}_{2} \mathrm{O}$ & $\mathrm{ScCl}_{3}$ & $2: 1$ & $\mathrm{SC}$ & 873 & $\times$ \\
\hline 0.33 & $\mathrm{Sc}_{2} \mathrm{MoO}_{6}$ & $\left(\mathrm{NH}_{4}\right)_{6} \mathrm{Mo}_{7} \mathrm{O}_{24} 4 \mathrm{H}_{2} \mathrm{O}$ & $\mathrm{ScCl}_{3}$ & $1: 2$ & $\mathrm{SC}$ & 873 & $\times$ \\
\hline 0.33 & $\mathrm{Sc}_{2} \mathrm{Ti}_{2} \mathrm{O}_{7}$ & $\mathrm{ScCl}_{3}$ & peroxo-Ti & $1: 1$ & $\mathrm{PC}$ & 1173 & - \\
\hline 0.32 & $\mathrm{Sc}_{2} \mathrm{Ti}_{2} \mathrm{O}_{7}$ & $\mathrm{ScCl}_{3}$ & peroxo-Ti & $1: 1$ & $\mathrm{PC}$ & 1073 & - \\
\hline 0.31 & $\mathrm{Sc}_{2} \mathrm{MoO}_{6}$ & $\left(\mathrm{NH}_{4}\right)_{6} \mathrm{Mo}_{7} \mathrm{O}_{24} 4 \mathrm{H}_{2} \mathrm{O}$ & $\mathrm{ScCl}_{3}$ & $1: 2$ & PC & 873 & - \\
\hline 0.30 & $\mathrm{~V}_{2} \mathrm{Mo}_{2} \mathrm{O}_{11}$ & $\mathrm{MoCl}_{5}$ & $\mathrm{NH}_{4} \mathrm{VO}_{3}$ & $1: 1$ & $\mathrm{SC}$ & 873 & $\checkmark$ \\
\hline 0.30 & $\mathrm{Sc}_{2} \mathrm{MoO}_{6}$ & $\left(\mathrm{NH}_{4}\right)_{6} \mathrm{Mo}_{7} \mathrm{O}_{24} 4 \mathrm{H}_{2} \mathrm{O}$ & $\mathrm{ScCl}_{3}$ & $1: 2$ & $\mathrm{SC}$ & 973 & - \\
\hline
\end{tabular}




\section{Supporting note.}

Given a pair of normalized histograms, $H$ and $I$, each containing $n$ bins, the histogram intersection (Swain, M. J. and Ballard, D. H., Colour indexing, Int. J. Comput. Vis. 1991, 7, 1132.) of them is defined as follows:

$$
H \cap I=\sum_{i=1}^{n} \min \left(H_{i}, I_{i}\right)
$$

